\title{
Risk of second cancer after initial treatment of breast cancer: An Osaka Cancer Registry Database study
}

\author{
YUMI KAMIGAKI $^{1}$ and KOJI KAWAKAMI ${ }^{2}$ \\ ${ }^{1}$ Department of Biostatistics, Kyoto University School of Public Health; ${ }^{2}$ Department of Pharmacoepidemiology, \\ Graduate School of Medicine and Public Health, Kyoto University, Kyoto, Japan
}

Received November 5, 2010; Accepted June 16, 2011

DOI: $10.3892 / \mathrm{ol} .2011 .339$

\begin{abstract}
In the present study, the association between the incidence of second cancer and initial treatment for primary breast cancer was investigated using the Osaka Cancer Registry in Japan. We selected 45,575 patients diagnosed with breast cancer between January 1975 and December 2003. Information on initial cancer treatment and second cancer was obtained from the Osaka Cancer Registry. Patients were classified according to initial treatment (chemo-, hormone, or radiotherapy, or no treatment). We calculated the incidence rate ratio of second cancers in patients classified by treatment for the first cancer. The effects of treatment adjusted for covariates on second cancers were examined using stratified analyses and a Cox proportional hazard model. The final number of 33,043 subjects had a mean duration of follow-up of $5.2 \pm 4.3$ years, during which 1,857 second cancers were diagnosed. For hormone therapy, the incidence rate ratio (IRR) of all second cancers was 0.64 [95\% confidence interval (CI), 0.58-0.70], and that of corpus uteri cancer was 3.04 (95\% CI, 1.78-5.19). The multivariate analysis revealed that the IRR of corpus uteri cancer associated with hormone therapy was 2.53 (95\% CI, 1.41-4.55). The incidence rate of all second cancers associated with initial treatment was lower than that associated with no treatment. Only second corpus uteri cancer may be related to hormone therapy.
\end{abstract}

\section{Introduction}

In Japanese women, the incidence of primary breast cancer, the most common cancer in women, is highest in those aged 40 to 50 years (1). The majority of patients undergo surgical resection, chemotherapy or radiotherapy. Although improvements in diagnostic and treatment techniques have increased the ratio of Japanese women surviving breast cancer (2), many

Correspondence to: Dr Koji Kawakami, Department of Pharmacoepidemiology, Graduate School of Medicine and Public Health, Kyoto University, Yoshidakonoe-cho, Sakyo-ku, Kyoto-shi, Kyoto 606-8507, Japan

E-mail: kawakami.koji.4e@kyoto-u.ac.jp

Key words: second cancer, breast cancer, Cancer Registry Database patients are at risk of developing second cancers. The acute side effects of various therapies have been well defined, but the subsequent complications of these therapies, including the development of second cancers, are less well known.

Second primary cancers are associated not only with the effects of cancer treatment but also with the effects of etiological factors common to first and second cancers, such as smoking, alcohol use and diet, as well as genetic, hormonal and environmental factors $(3,4)$. Risk is also likely associated with genetic variation. Cancer patients may also experience various chemotherapy- or radiation-induced DNA double strand break-related gene translocations and genomic instability conferred by the loss of DNA repair (5-7). These mechanisms have been reported to play a role in the possible development of second cancers. With regard to chemotherapy, a number of reports suggested an association between second cancers and agents such as cisplatin, topoisomerase inhibitors and methylating agents. Tamoxifen citrate is often administered as hormone therapy in breast cancer patients However, various studies have revealed that tamoxifen causes endometrial cancer (8-11).

Concerning the time of occurrence of second cancers, data from patients treated for Hodgkin's lymphoma revealed that risk was generally highest at 15-20 years after radiotherapy, and decreased only slightly following this period of time (12). In contrast, the risk of developing acute myeloid lymphoma (AML) was highest considerably earlier, at 2-7 years after chemotherapy, and decreased after this period of time (13). Given that the complications of cancer treatment are rare and delayed, the tracing and studying of second cancers would be facilitated by a large, long-term database.

Data pertaining to the risk of second cancers after breast cancer from European and US cancer registries have been published (14-16). The most common second cancer identified was endometrial cancer. However, a wide variety of other sites were identified, including the ovary, thyroid gland, lung and blood/bone marrow.

Results for cancer registry studies on second cancer in Japan have been ambiguous. In their study, Murakami and colleagues reported that common second cancer sites were the stomach, colon and thyroid gland (17). However, this study was published in 1987, prior to the approval of tamoxifen citrate in Japan. Using cancer registry data from a hospital in Osaka, Tanaka and colleagues reported that the 


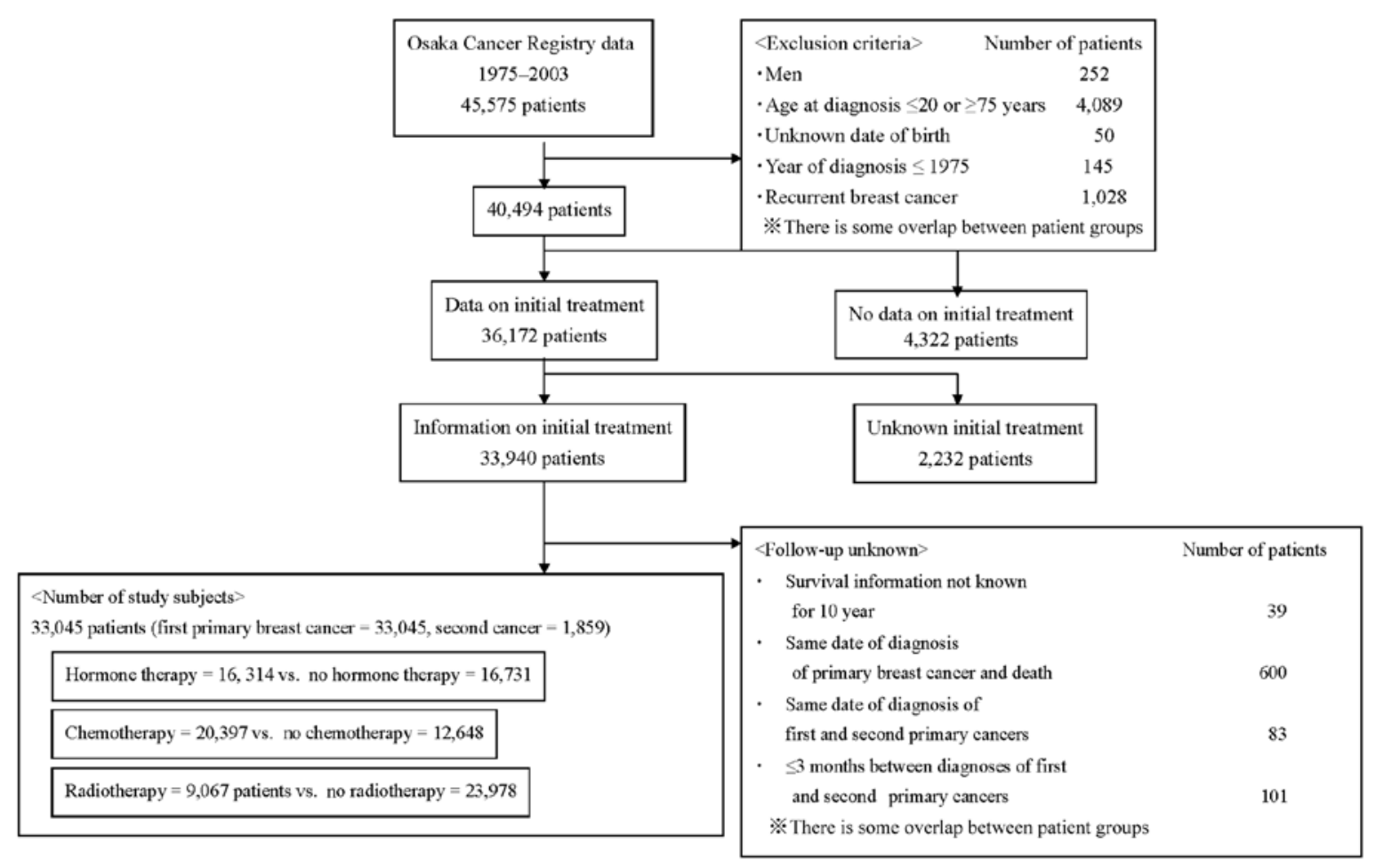

Figure 1. Flow chart showing selection of study subjects.

most common sites of second cancer after breast cancer were the ovary, thyroid gland and lymphocytes (non-Hodgkin's lymphoma) (18). However, this study analyzed data from only one hospital. Moreover, findings of previous studies using the Osaka Cancer Registry database showed no increase in the risk of second corpus uteri cancer after hormonal treatment of primary breast cancer. Etiological factors in Japan differ from those in Europe and the United States, and long-term data from these countries are not applicable to Japanese patients, highlighting the importance of investigating the incidence of second cancers over time in Japan.

In the present study, we used the Osaka Cancer Registry to retrospectively investigate the association between the incidence of second cancers and initial treatment (chemotherapy, hormone therapy and radiotherapy) in breast cancer patients.

\section{Patients and methods}

Patients. The subjects were 45,575 individuals who had been diagnosed with breast cancer between January 1975 and December 2003 (Fig. 1). Of these individuals, exclusion criteria were applied to 252 men; 4,089 patients $\leq 20$ or $\geq 75$ years of age at the time of diagnosis; 145 diagnosed with primary breast cancer prior to January 1975; and 1,028 registered as having recurrent breast cancer. We then excluded 50 patients whose date of birth was unknown; 4,322 without data on initial treatment for primary breast cancer and 2,232 with incomplete data; 600 for whom the first primary cancer diagnosis and death were recorded simultaneously and 83 for whom the first and second primary cancers were diagnosed on the same date; 101 for whom the time between the first and second primary cancer diagnoses was $\leq 3$ months; and 39 for whom survival information for $\geq 10$ years after diagnosis of the primary breast cancer was unknown. Finally, 33,045 primary breast cancer patients were enrolled in the study.

Data collection. Information on treatment of the initial and second cancer was obtained for each patient using ICD-10-coded data in the Osaka Cancer Registry. ICD-10 coding of second cancers follows common rules set by the International Association Cancer Registry and International Agency for Research on Cancer, which state that a primary cancer is one that originates from a primary site or tissue. Second breast cancer is defined as having different histology to the primary breast cancer: extension, recurrence, and metastasis are not considered second cancers.

Second cancers occurred in the pharynx, esophagus, stomach, small intestine, colon, rectum, liver, gallbladder and bile duct, pancreas, lung, breast, cervix, corpus uteri, ovary, kidney, bladder, brain, thyroid, skin, lymphocytes, and myelocytes (in some cases manifesting as AML). In this study, second cancers of the cervix or corpus uteri were not included when they were described as not otherwise specified. In addition, no case of carcinoma in situ was included. If a breast cancer had different Berg histologic types, it was recorded as a multiple cancer. Furthermore, the definition of second breast cancer differed from that of so-called contralateral breast cancer. The following variables were included: age, gender, date of diagnosis of first primary cancer, diagnosis of first primary cancer (ICD-10), diagnosis of second primary cancer (ICD-10), date of diagnosis of second primary cancer, and initial treatment, defined as that administered within 4 months of the diagnosis of primary breast cancer or already planned for primary breast cancer. The following variables were not 
included: date on which the patient was no longer included in the registry, information on menopausal status at diagnosis of breast primary cancer and estrogen status. Information on initial treatment did not include the names of any drugs administered, their dosage or duration of administration. This study was approved by the medical ethics committee of Kyoto University.

Statistical analysis. Patients were classified according to the initial treatment received (chemotherapy, hormone therapy, radiotherapy or no treatment). In each treatment group, the incidence rate for each type of second cancer was estimated as the observed number of events divided by the total number of person-years at risk. The incidence rate ratios (IRR) between the treatment and corresponding non-treated group were then compared. In these calculations, patients were considered to be at risk from the date of diagnosis of the primary breast cancer until the date of diagnosis of the respective second cancer, date of death, last date on which the patient was known to be alive, or end of the study period (December 31, 2003), whichever criterion occurred first. The effects of treatment on the second cancer were adjusted for covariates and analyzed with stratification by age at the first primary breast cancer, time since diagnosis of the first primary breast cancer, and type of combined treatment. The risk of corpus uteri cancer was evaluated using a Cox proportional hazard model adjusted for other covariates, such as age at diagnosis, chemotherapy, radiotherapy, and lymph node metastasis. Statistical analyses were performed using SAS v. 9.1 (SAS Institute, Inc.; Cary, NC, USA) and JMP 7 (SAS Institute, Inc.).

\section{Results}

Patient characteristics. A total of 33,045 patients were enrolled. Background characteristics are shown in Table I. Due to coding errors, the location of the second cancer was not verified in 2 patients. Thus, these 2 patients were excluded, leaving 33,043 study subjects for analysis. Of the 33,045 study subjects, 16,314 received hormone therapy $(49.36 \%)$, whereas $16,731 \mathrm{did}$ not (50.63\%); 20,397 received chemotherapy (61.72\%) whereas 12,648 did not (38.28\%); and 9,067 received radiotherapy (27.44\%), whereas 23,978 did not $(72.56 \%)$.

With regard to hormone therapy, distribution by age at diagnosis, stage of primary breast cancer, and time since first primary breast cancer occurred was similar between those who did and did not receive hormone therapy. By contrast, a difference was observed in the year of diagnosis.

For chemotherapy, distribution by age at diagnosis was similar between the groups. Of the chemotherapy-treated patients, $44.2 \%$ presented lymph node metastasis compared with $20.3 \%$ in the non-chemotherapy-treated group. Year of diagnosis, stage of primary breast cancer, and time since first primary breast cancer occurred differed between the groups.

For radiotherapy, distribution by age at diagnosis was similar between the groups. Of the radiotherapy-treated patients, $44.2 \%$ had lymph node metastasis compared with $20.3 \%$ in the non-radiotherapy-treated patients. Year of diagnosis, stage of primary breast cancer, and time since first primary breast cancer occurred differed between the groups.
Hormone therapy versus no hormone therapy. Table II shows the number and frequency of second cancers and compares the IRR between patients who did and did not receive hormone therapy. The total number of second primary cancers in those treated with hormones was 699 versus 1,158 in those who were not treated with hormones. The incidence rate of all second cancers per 1,000 women-years was 8.2 in the hormone therapy group and 12.9 in the non-hormone therapy group. IRR was 0.64 [95\% confidence interval (CI), 0.58-0.70]. IRR for corpus uteri cancer was 3.04 (95\% CI, 1.78-5.19), while those for other types of second cancers were less than one. With regard to the site of second cancers, IRR was statistically significantly lower in the case of the colon (IRR 0.52 , 95\% CI, 0.38-0.71), liver (IRR =0.67, 95\% CI, 0.46-0.98), and pancreas (IRR $=0.56,95 \%$ CI, 0.34-0.93). Similar results were obtained even when the 17 patients with in situ corpus uteri carcinoma were included in the IRR calculation (data not shown).

For many cancer sites, risk increased with an increase in age at diagnosis of the first primary breast cancer (Table III). Although IRR of the corpus uteri was $1.36(\mathrm{P}=0.83)$ for patients aged 20-44 years at diagnosis of the first primary breast cancer, that for patients aged $>45$ years was $3-4$, with these IRRs being significantly high.

For corpus uteri cancer, IRR was 2.10 (95\% CI, 0.91-23.12) within 1 year of diagnosis, 2.49 (95\% CI, 0.89-6.97) at 1-4 years after diagnosis, $3.65(95 \% \mathrm{CI}, 1.51-8.82)$ at 5-9 years after diagnosis, and 2.07 (95\% CI, 0.75-5.70) at $\geq 10$ years after diagnosis. IRR of corpus uteri cancer was therefore highest at 5-9 years after breast cancer diagnosis. For stomach and ovary cancers, risk was most pronounced within the first year of follow-up (Table IV).

Risk for corpus uteri cancer was relatively constant over time, at 3.44 (95\% CI, 0.31-37.88) in 1975-1981, 2.74 (95\% CI, 1.13-6.65) in 1982-1988, 2.87 (95\% CI, 1.18-6.98) in 1989-1995, and 1.85 (95\% CI, 0.51-6.71) in 1989-2003.

Chemotherapy versus no chemotherapy. Table V shows the number and frequency of second cancers and a comparison of IRRs between patients who did and did not receive chemotherapy. The total number of second primary cancers was 1,180 in the chemotherapy-treated versus 677 in the non-chemotherapy-treated patients. IRR was 0.91 (95\% CI, 0.83-1.00). IRRs of chemotherapy vs. no chemotherapy were significantly higher for cancer of the corpus uteri (IRR $=2.32,95 \% \mathrm{CI}, 1.27-4.23$ ), and gallbladder and bile duct (IRR $=1.82,95 \% \mathrm{CI}, 1.01-3.30)$. By contrast, IRRs were significantly lower for the liver $(\mathrm{IRR}=0.54$, 95\% CI, 0.37-0.78) and kidney (IRR $=0.36,95 \% \mathrm{CI}, 0.15$ 0.84). On stratification by treatment, the risk of AML in the chemotherapy-only group was 4.17 (95\% CI, 0.91-19.04) (data not shown).

Radiotherapy versus no radiotherapy. Table VI compares IRRs between patients who received radiotherapy and those who did not. The total number of second primary cancers was 1,428 in patients treated with radiotherapy versus 429 in those who were not. IRR was 0.97 (95\% CI, 0.87-1.08). IRR associated with radiotherapy versus no radiotherapy for second breast cancer was 1.46 (95\% CI, 1.13-1.90), whereas that for small intestine 


\begin{tabular}{|c|c|c|c|c|c|}
\hline 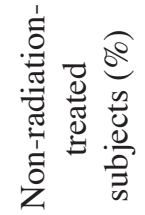 & 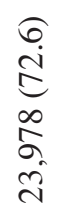 & 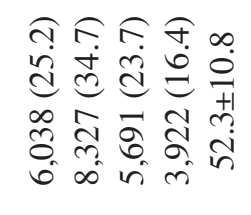 & 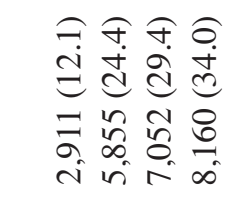 & 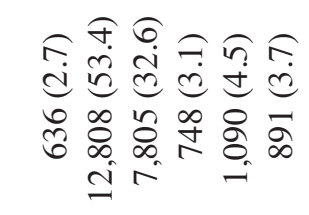 & 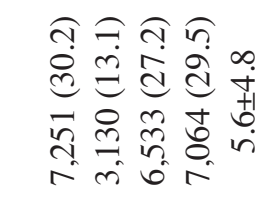 \\
\hline 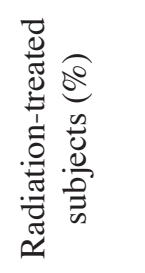 & 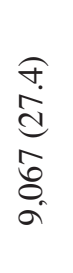 & 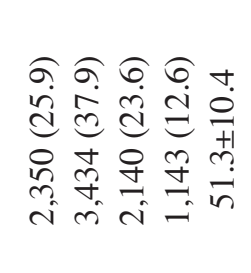 & 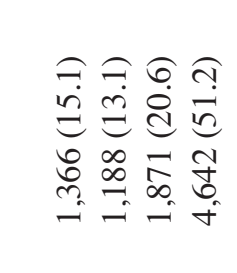 & 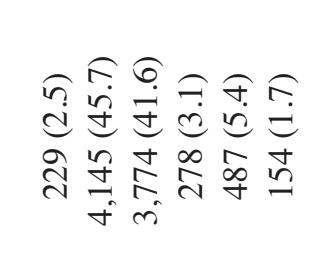 & 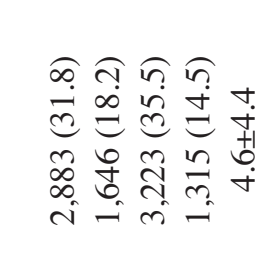 \\
\hline 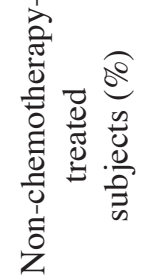 & 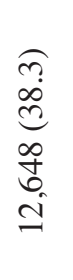 & 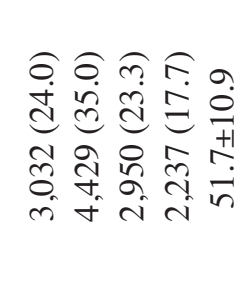 & 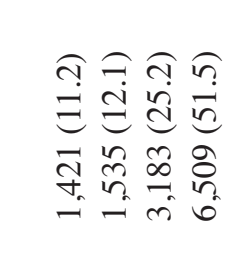 & 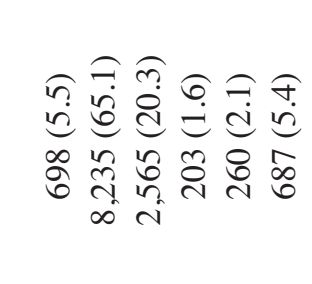 & 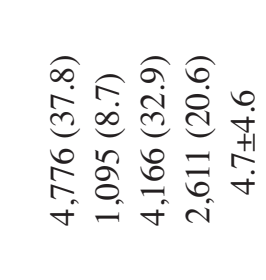 \\
\hline 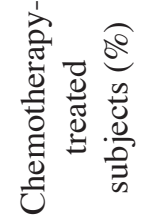 & 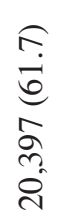 & 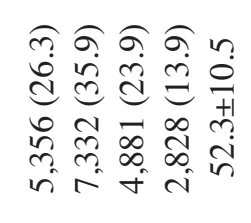 & 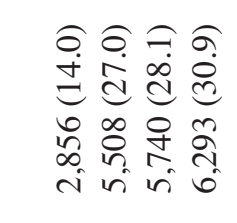 & 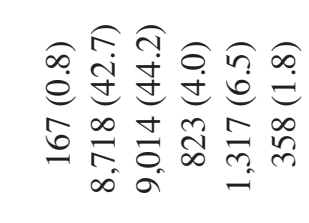 & 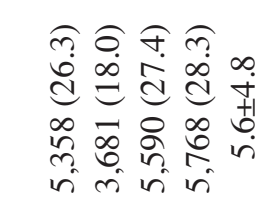 \\
\hline 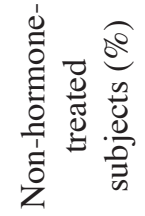 & 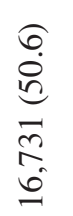 & 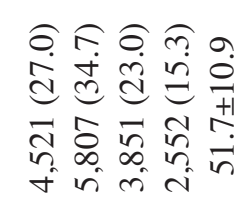 & 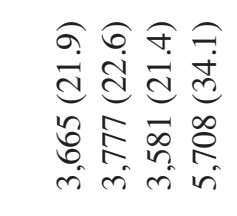 & 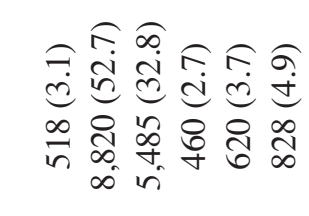 & 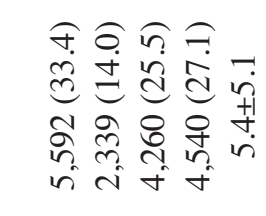 \\
\hline 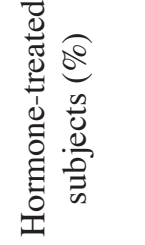 & 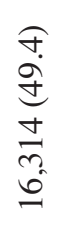 & 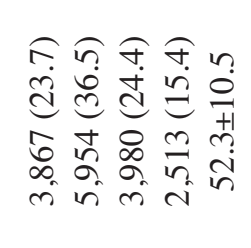 & 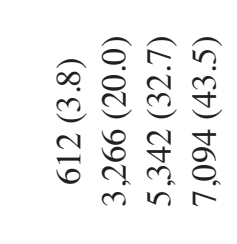 & 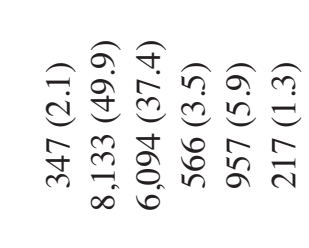 & 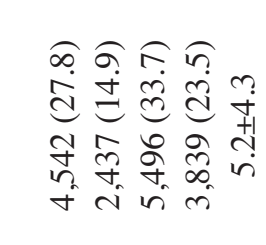 \\
\hline 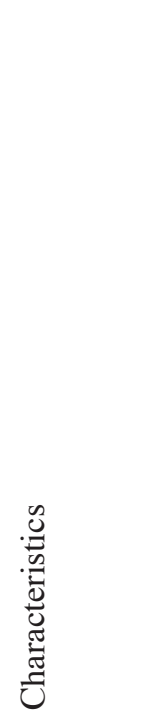 & & 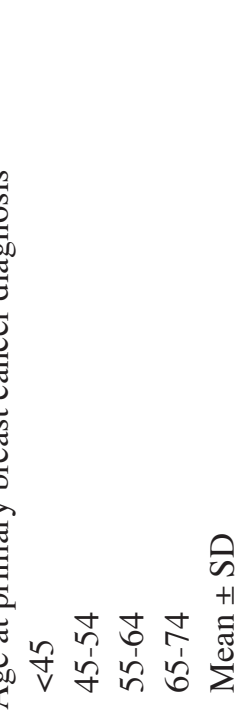 & 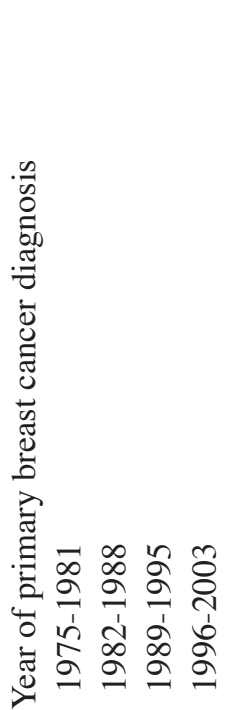 & 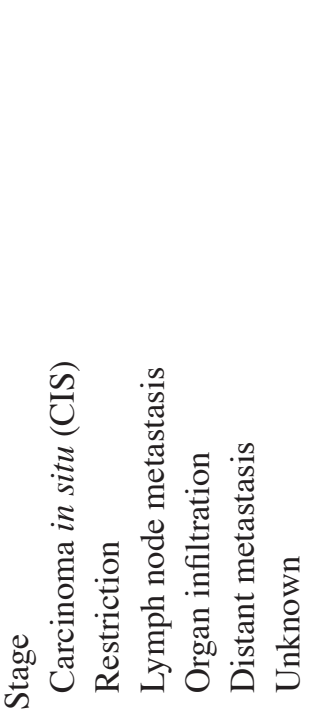 & 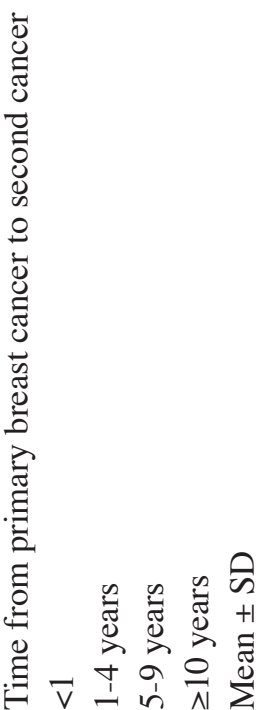 \\
\hline
\end{tabular}




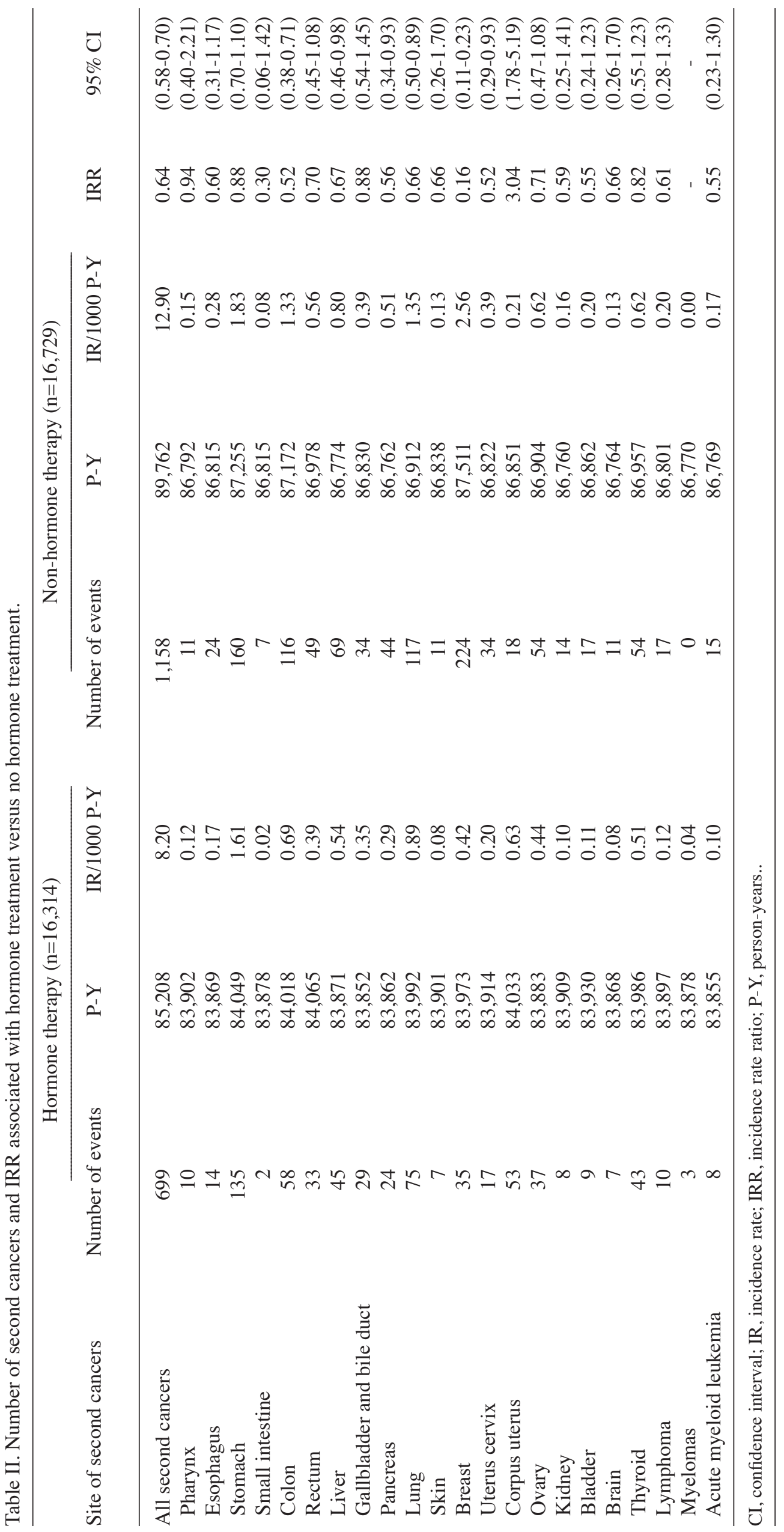




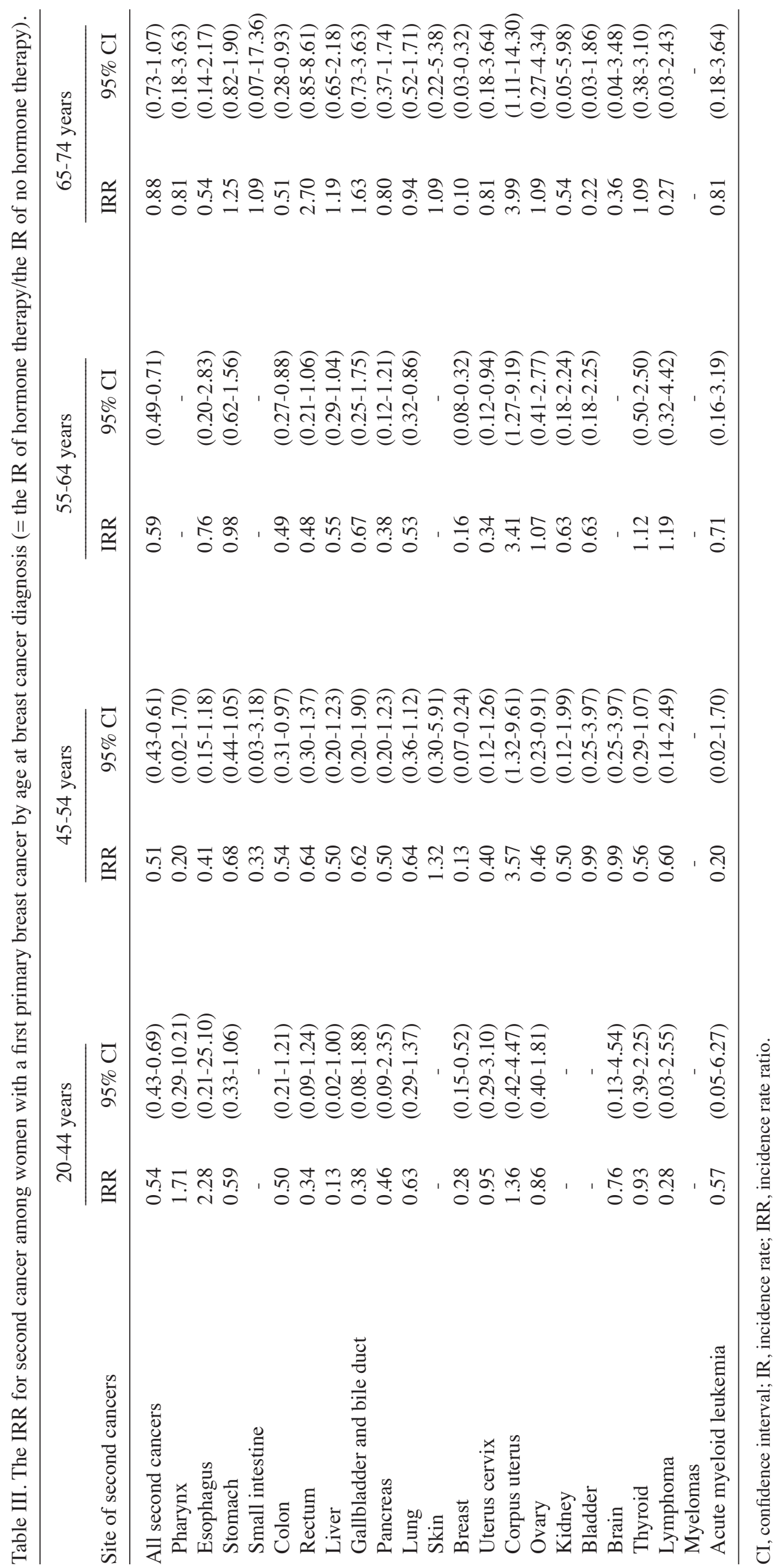




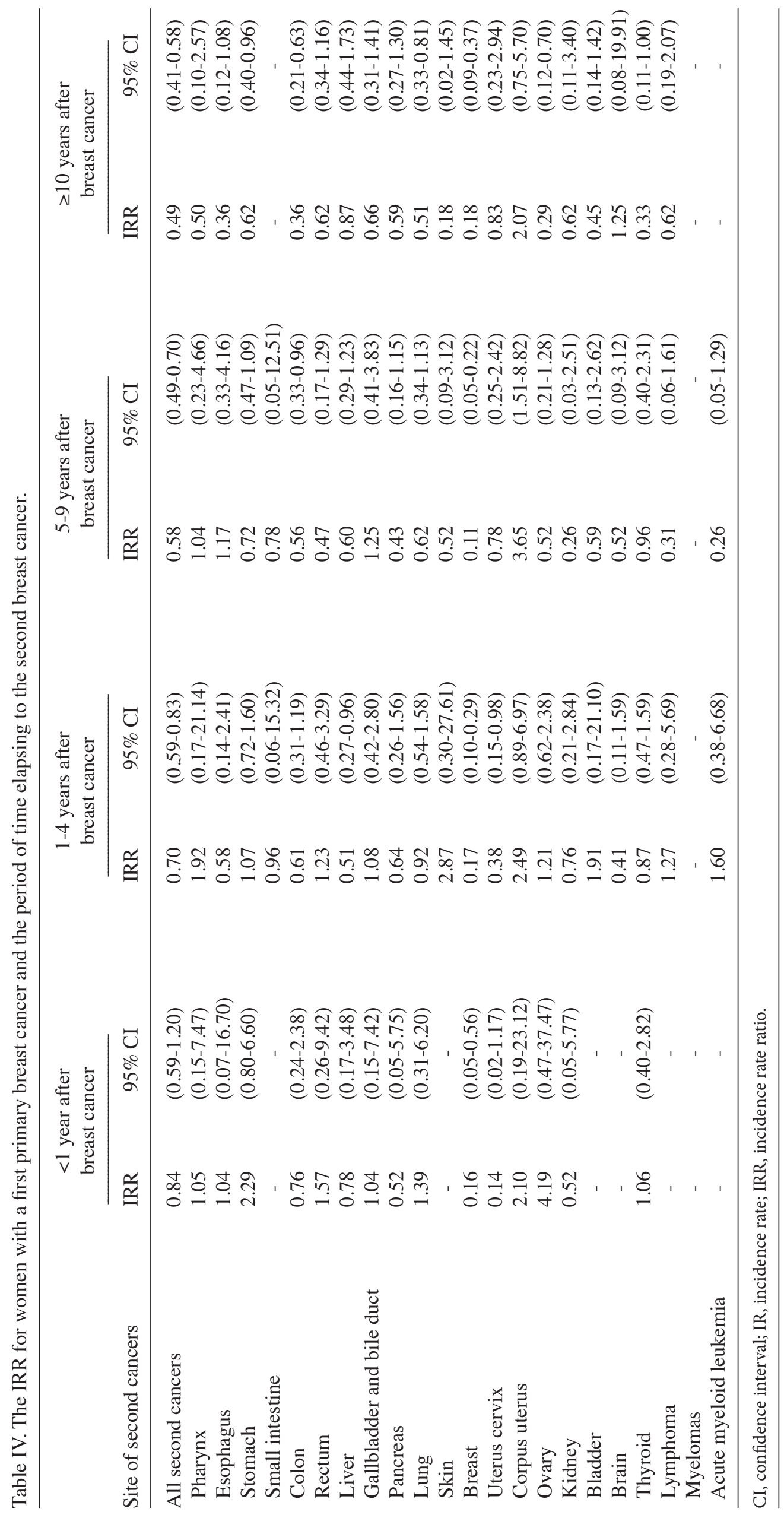




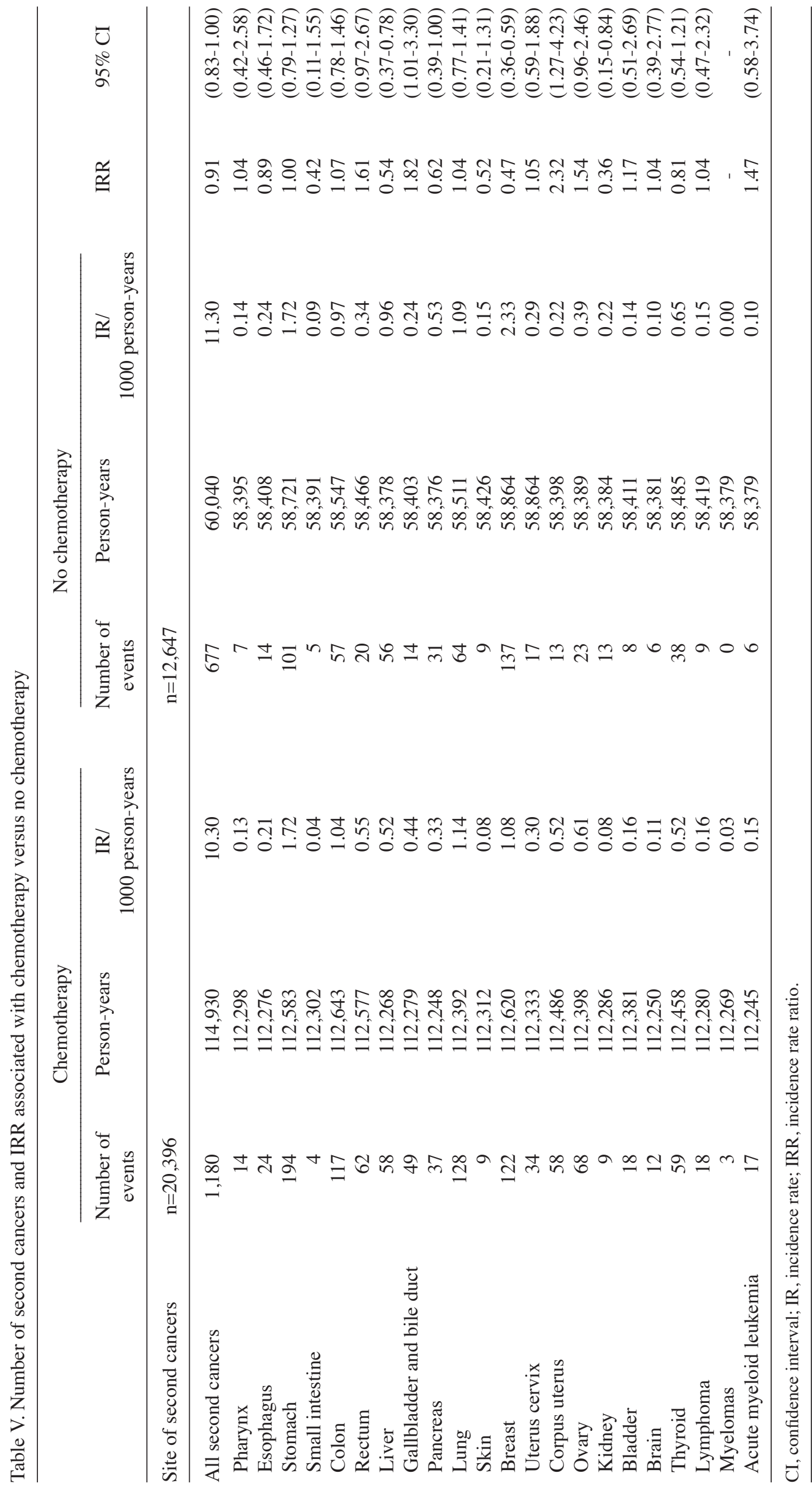


כ0

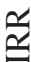

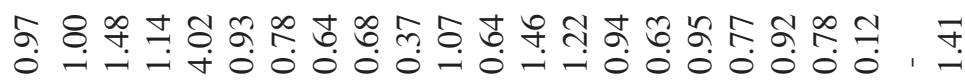

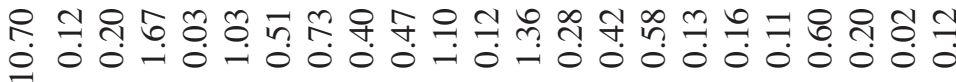

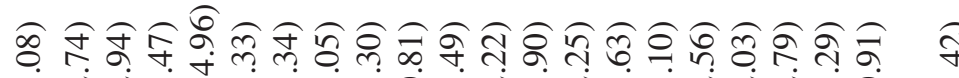

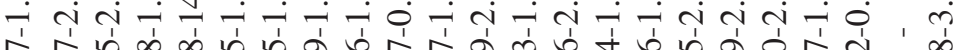

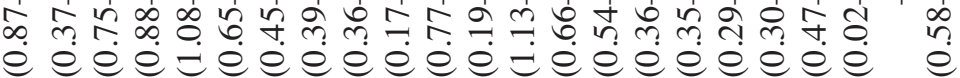

హㅇำ ল্র

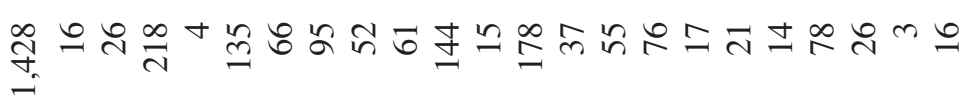
है II

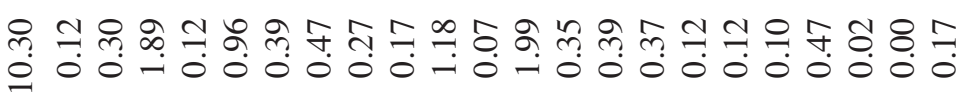

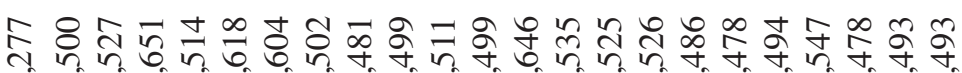

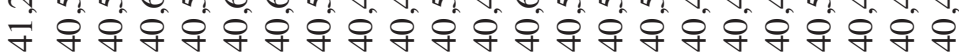
$\stackrel{0}{0}$ 范

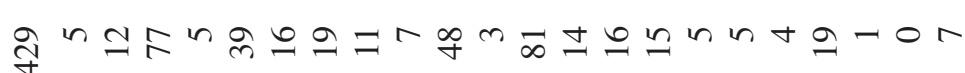
离 (1)

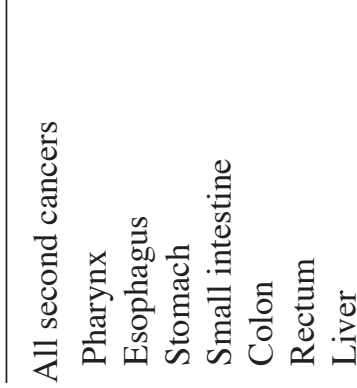


cancer was 4.02 (95\% CI, 1.08-14.96). IRRs were significantly lower for lymphoma (IRR $=0.12,95 \%$ CI, 0.02-0.91). On stratification by treatment, the results exhibited a similar tendency to those for hormone therapy (data not shown).

Multivariate analysis. The effect of hormone therapy adjusted for covariates on corpus uteri cancer was examined by using the Cox proportional hazard model. For second corpus uteri cancer, the IRR associated with hormone therapy after covariate adjustment was 2.53 (95\% CI, 1.41-4.55) (Table VII).

\section{Discussion}

A large-scale retrospective study was conducted using the Osaka Cancer Registry in Japan to investigate the risk of second primary cancers after first primary breast cancer. Of 33,045 female individuals with first primary breast cancer, 1,859 developed second cancers. Of these, the IRR of corpus uteri cancer was 3.04 (95\% CI, 1.78-5.19) for hormone therapy. Although IRRs of all second cancers associated with initial cancer treatment were low, these findings may indicate an association between second corpus uteri cancer and hormone therapy.

Following its approval in Japan in 1982, tamoxifen citrate assumed a central role in hormone therapy. However, long-term research on this drug is currently lacking. In our study, second corpus uteri cancer was strongly related to hormone therapy for first primary breast cancer. Previous adjuvant trials and observation studies reported that the relative risk of developing endometrial cancer is 2- or 3-fold higher after tamoxifen citrate treatment (19-21). The findings of the present study revealed that the risk was high even prior to 1975 , when tamoxifen citrate was rarely used in Japan. This increase in risk is likely associated with a number of common risk factors, such as reproductive and genetic factors, obesity, and other factors that have yet to be identified (6). Furthermore, we also observed that the risk of developing second cancer was higher for women whose age at diagnosis of first breast cancer was 45 years or older. Age may clearly be a surrogate of susceptibility to cancer and immune response. Notably, we observed that the IRR of corpus uteri cancer was highest at 5-9 years after first breast cancer diagnosis, indicating the necessity of monitoring for this cancer within the first 10 years of breast cancer diagnosis. Nevertheless, the proportion of women who develop corpus uteri cancer is markedly low compared with that of women who benefit from the drug.

With regard to chemotherapy, various regimens have been used over time, depending on the stage of breast cancer. In the present study, the IRR for AML, suggested to be the main type of second cancer associated with chemotherapy (22), was found to be 1.47 within the follow-up time, and stratification by treatment revealed a risk of AML in the chemotherapy-only group of 4.17 (95\% CI, 0.91-19.04). An association of AML with exposure to alkylating agents, such as cyclophosphamide and topoisomerase II inhibitors, such as epirubicin, has been described (23-27). Thus, we observed a decreased incidence of second cancer for liver and kidney cancers. Although certain chemotherapeutic drugs have been reported to prevent carcinogenesis or cancer promotion in vitro or in animal models $(28,29)$, the present results did not indicate which drugs for breast cancer may prevent lung or renal cell carcinogenesis. Our study also revealed that the risk of corpus uteri cancer
Table VII. Analyses for the incidence of the corpus uterus cancer.

\begin{tabular}{lcc}
\hline & Hazard ratio & $95 \%$ CI \\
\hline Hormone therapy (yes/no) & 2.53 & $(1.41-4.55)$ \\
Radiotherapy (yes/no) & 1.12 & $(0.63-2.01)$ \\
Chemotherapy (yes/no) & 1.74 & $(0.91-3.33)$ \\
Age (per decade) & 1.40 & $(1.31-1.42)$ \\
Lymph node metastasis (yes/no) & 0.99 & $(0.60-1.62)$ \\
\hline
\end{tabular}

CI, confidence interval.

was high in those receiving versus not receiving chemotherapy. Evaluation of the effect of hormone therapy adjusted for covariates on corpus uteri cancer by stratified analysis and a Cox proportional hazard model revealed that the IRR of second corpus uteri cancer was significant for patients who received hormone therapy, but was not significant for those who did not.

For radiotherapy, the most common second cancer types were those of organs located close to the breast, such as the esophagus, lung, thyroid gland and stomach, as well as soft tissue sarcomas of the thorax and upper limbs and leukemia (30-36). Among radiotherapy-treated patients, certain investigators have reported an increase in the risk of contralateral breast cancer associated with radiotherapy $(37,38)$. In the present study, primary breast cancer was diagnosed as a second breast cancer if it had a different tissue diagnosis to the first cancer, whether it was contralateral or not. The present study revealed that the risk of second breast cancer with different pathological features was also higher in those receiving radiotherapy. Of note is that second breast cancer in this study was different to contralateral breast cancer. We noted an excess incidence of cancer of the small intestine in this study, but the incidence of second small intestinal cancer was markedly low. Thus, further studies should be conducted with regard to this observation. Although a decrease was noted in the incidence of lymphoma in this study, this association has been observed in few previous studies.

Our results should be considered within the limitations of cancer registry-based data. Population-based studies allow for a better evaluation of site-specific second cancer risks among numerous patients than hospital-based studies. However, such studies do not allow for the evaluation of significant cancerrelated factors such as smoking, alcohol consumption, her 2 or family history. Although we focused on initial treatment as the primary explanation for the observed secondary cancers, our results should be interpreted with care, as risk may also be affected by family history- and etiology-related factors.

A second limitation of this study is that the treatment data pertained only to the initial treatment, which was recorded as the first-line treatment plan within 4 months from the diagnosis of primary breast cancer. Hormone therapy data may be more reliable than data for chemotherapy and radiotherapy, since most doctors measure estrogen receptor levels and consider the use of hormone therapy when breast cancer is diagnosed.

In conclusion, the IRR of all second cancers associated with initial cancer treatment was low. Among all treatments for first cancer, the incidence of second corpus uteri cancer was found to be possibly related to hormone therapy. 


\section{Acknowledgements}

We thank Hideaki Tsukuma and Takashi Omori for their valuable suggestions, and the Osaka Cancer Registry for providing access to registry data.

\section{References}

1. Minami Y, Tsubono Y, Nishino Y, Ohuchi N, Shibuya D and Hisamichi S: The increase of female breast cancer incidence in Japan: emergence of birth cohort effect. Int J Cancer 108: 901-906, 2004

2. Tsukuma H, Ajiki W, Ioka A and Oshima A: Research Group of Population-Based Cancer Registries of Japan. Survival of cancer patients diagnosed between 1993 and 1996: a collaborative study of population-based cancer registries in Japan. Jpn J Clin Oncol 36: 602-607, 2006

3. Knight JA, Bernstein L, Largent J, et al: WECARE Study Collaborative Group, Bernstein JL. Alcohol intake and cigarette smoking and risk of a contralateral breast cancer: The Women's Environmental Cancer and Radiation Epidemiology Study. Am J Epidemiol 169: 962-968, 2009.

4. Sánchez L, Lana A, Hidalgo A, et al: Risk factors for second primary tumours in breast cancer survivors. Eur $\mathrm{J}$ Cancer Prev 17: 406-413, 2008.

5. Travis LB, Andersson M, Gospodarowicz M, et al: Treatmentassociated leukemia following testicular cancer. J Natl Cancer Inst 92: 1165-1171, 2000.

6. Allan JM and Travis LB: Mechanisms of therapy-related carcinogenesis. Nat Rev Cancer 12: 943-955, 2005.

7. Travis LB, Holowaty EJ, Bergfeldt K, et al: Risk of leukemia after platinum-based chemotherapy for ovarian cancer. N Engl J Med 340: 351-357, 1999.

8. Early Breast Cancer Trialists' Collaborative Group: Tamoxifen for early breast cancer: an overview of the randomised trials. Lancet 351: 1451-1467, 1998.

9. Swerdlow AJ and Jones ME: Tamoxifen treatment for breast cancer and risk of endometrial cancer, a case-control study. J Natl Cancer Inst 97: 375-384, 2005.

10. Yamazawa K, Miyazawa Y, Suzuki M, Wakabayashi M, Kaku H, Matsui $\mathrm{H}$ and Sekiya S: Tamoxifen and the risk of endometrial cancer in Japanese women with breast cancer. Surg Today 36 41-46, 2006.

11. Matsuyama Y, Tominaga T, Nomura Y, et al: Second cancers after adjuvant tamoxifen therapy for breast cancer in Japan. Ann Oncol 11: 1537-1543, 2000.

12. Travis LB, Hill DA, Dores GM, et al: Breast cancer following radiotherapy and chemotherapy among young women with Hodgkin disease. JAMA 290: 465-475, 2003.

13. Dores GM, Metayer C, Curtis RE, et al: Second malignant neoplasms among long-term survivors of Hodgkin's disease: a population-based evaluation over 25 years. J Clin Oncol 20: 3484-3494, 2002.

14. Mellemkjaer L, Friis S, Olsen JH, et al: Risk of second cancer among women with breast cancer. Int J Cancer 118: 2285-2292, 2006.

15. Cluze C, Delafosse P, Seigneurin A and Colonna M: Incidence of second cancer within 5 years of diagnosis of a breast, prostate or colorectal cancer: a population-based study. Eur J Cancer Prev 18: 343-348, 2009

16. Kirova YM, de Rycke Y, Gambotti L, Pierga JY, Asselain B and Fourquet A. Institut Curie Breast Cancer Study Group: Second malignancies after breast cancer: the impact of different treatment modalities. Br J Cancer 98: 870-874, 2008.

17. Murakami R, Hiyama T, Hanai A and Fujimoto I: Second primary cancers following female breast cancer in Osaka, Japan - a population-based cohort study. Jpn J Clin Oncol 17: 293-302, 1987.

18. Tanaka H, Tsukuma H, Koyama H, Kinoshita Y, Kinoshita N and Oshima A: Second primary cancers following breast cancer in the Japanese female population. Jpn J Cancer Res 92: 1-8, 2001.

19. Peters-Engl C, Frank W, Danmayr E, Friedl HP, Leodolter S and Medl M: Association between endometrial cancer and tamoxifen treatment of breast cancer. Breast Cancer Res Treat 54: 255-260, 1999.
20. Van Leeuwen FE, Benraadt J, Coebergh JW, et al: Risk of endometrial cancer after tamoxifen treatment of breast cancer. Lancet 343: 448-452, 1994.

21. Fisher B, Costantino JP, Redmond CK, Fisher ER, Wickerham DL and Cronin WM: Endometrial cancer in tamoxifen-treated breast cancer patients: findings from the National Surgical Adjuvant Breast and Bowel Project (NSABP) B-14. J Natl Cancer Inst 86: 527-537, 1994.

22. Howard RA, Gilbert ES, Chen BE, et al: Leukemia following breast cancer: an international population-based study of 376,825 women. Breast Cancer Res Treat 105: 359-368, 2007.

23. Pedersen-Bjergaard J, Sigsgaard TC, Nielsen D, et al: Acute monocytic or myelomonocytic leukemia with balanced chromosomal translocations to band 11q23 after therapy with 4-epidoxorubicin and cisplatin or cyclophosphamide for breast cancer. J Clin Oncol 10: 1444-1515, 1992.

24. Smith RE: Risk for the development of treatment-related acute myelocytic leukemia and myelodysplastic syndrome among patients with breast cancer: review of the literature and the National Surgical Adjuvant Breast and Bowel Project experience. Clin Breast Cancer 4: 273-279, 2003.

25. Smith RE, Bryant J, DeCillis A and Anderson S: Acute myeloid leukemia and myelodysplastic syndrome after doxorubicincyclophosphamide adjuvant therapy for operable breast cancer: the National Surgical Adjuvant Breast and Bowel Project Experience. J Clin Oncol 21: 1195-1204, 2003.

26. Tallman MS, Gray R, Bennett JM, et al: Leukemogenic potential of adjuvant chemotherapy for early-stage breast cancer: the Eastern Cooperative Oncology Group experience. J Clin Oncol 13: 1557-1563, 1995.

27. Crump M, Tu D, Shepherd L, Levine M, Bramwell V and Pritchard K: Risk of acute leukemia following epirubicin-based adjuvant chemotherapy: a report from the National Cancer Institute of Canada Clinical Trials Group. J Clin Oncol 21: 3066-3071, 2003.

28. Satyamoorthy K and Perchellet JP: Inhibition of mouse skin tumor promotion by adriamycin and daunomycin in combination with verapamil or palmitoylcarnitine. Cancer Lett 55: 135-142, 1990.

29. Wattenberg LW, Wiedmann TS and Estensen RD: Chemoprevention of cancer of theupper respiratory tract of the Syrian golden hamster by aerosol administration ofdifluoromethylornithine and 5-fluorouracil. Cancer Res 64: 2347-2349. 2004

30. Andersson M, Jensen MB, Engholm G and Storm HH: Risk of second primary cancer among patients with early operable breast cancer registered or randomised in Danish Breast Cancer cooperative Group (DBCG) protocols of the 77, 82 and 89 programmes during 1977-2001. Acta Oncol 47: 755-764. 2008.

31. Schaapveld M, Visser O, Louwman MJ, et al: Risk of new primary nonbreast cancers after breast cancer treatment: a Dutch population-based study. J Clin Oncol 26: 1239-1246, 2008.

32. Galper S, Gelman R, Recht A, et al: Second nonbreast malignancies after conservative surgery and radiation therapy for early-stage breast cancer. Int J Radiat Oncol Biol Phys 52: 406-414, 2002.

33. Fowble B, Hanlon A, Freedman G, Nicolaou N and Anderson P: Second cancers after conservative surgery and radiation for stages I-II breast cancer: identifying a subset of women at increased risk. Int J Radiat Oncol Biol Phys 51: 679-690, 2001.

34. Rubino C, de Vathaire F, Diallo I, Shamsaldin A and Lê MG: Increased risk of second cancers following breast cancer: role of the initial treatment. Breast Cancer Res Treat 61: 183-195, 2000.

35. Brown LM, Chen BE, Pfeiffer RM, et al: Risk of second nonhematological malignancies among 376,825 breast cancer survivors. Breast Cancer Res Treat 106: 439-451, 2007.

36. Kirova YM, Gambotti L, de Rycke Y, Vilcoq JR, Asselain B and Fourquet A: Risk of second malignancies after adjuvant radiotherapy for breast cancer: a large-scale, single-institution review. Int J Radiat Oncol Biol Phys 68: 359-363, 2007.

37. Bernstein JL, Haile RW, Stovall M, et al: WECARE Study Collaborative Group. Radiation exposure, the ATM Gene, and contralateral breast cancer in the women's environmental cancer and radiation epidemiology study. J Natl Cancer Inst 102: 475-483. 2010

38. Stovall M, Smith SA, Langholz BM, et al: Dose to the contralateral breast from radiotherapy and risk of second primary breast cancer in the WECARE study. Int J Radiat Oncol Biol Phys 72: 1021-1030, 2008. 